\title{
Multifrequency spatial filtering: A general property of two-dimensional photonic crystals
}

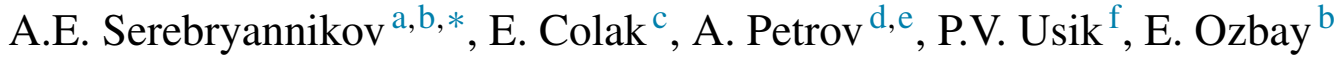 \\ ${ }^{a}$ Faculty of Physics, Adam Mickiewicz University, 61-614 Poznań, Poland \\ ${ }^{\mathrm{b}}$ Nanotechnology Research Center - NANOTAM, Bilkent University, 06800 Ankara, Turkey \\ ${ }^{\mathrm{c}}$ Department of Electrical and Electronics Engineering, Ankara University, Golbasi, 06830 Ankara, Turkey \\ ${ }^{\mathrm{d}}$ Institute of Optical and Electronic Materials, Hamburg University of Technology, 21073 Hamburg, Germany \\ ${ }^{\mathrm{e}}$ ITMO University, 49 Kronverskii Ave., 197101 St. Petersburg, Russia \\ ${ }^{\mathrm{f}}$ Institute of Radio Astronomy, National Academy of Sciences of Ukraine, 61002 Kharkiv, Ukraine
}

Received 23 March 2015; received in revised form 5 October 2015; accepted 19 November 2015

Available online 28 November 2015

\begin{abstract}
Spatial filtering, an analog of frequency-domain filtering that can be obtained in the incidence angle domain at a fixed frequency is studied in the transmission mode for slabs of two-dimensional rod-type photonic crystals. In the present paper, the emphasis is put on the demonstration of the possibility to obtain various regimes of spatial filtering, i.e., band-stop, band-pass, and low-pass filtering in different frequency ranges in one simple configuration. The operation is based on the use of several Floquet-Bloch modes with appropriate dispersion properties, so that such one or two co-existing mode(s) contribute to the forming of a proper filter characteristic within each specific frequency range. It is shown that high-efficiency transmission and steep switching between pass and stop bands can be obtained in the angle domain for wide ranges of variation of the problem parameters. In particular, by varying the rod-diameter-to-lattice-constant ratio, one attains lots of freedom in the engineering of spatial filters with desired transmission characteristics.
\end{abstract}

(C) 2015 Elsevier B.V. All rights reserved.

Keywords: Spatial filtering; Photonic crystal; Floquet-Bloch mode; Transmission; Fabry-Perot resonance

\section{Introduction}

Spatial (angular) filters are important components required in information processing and image enhancement for various frequency ranges. They operate in the incidence angle domain at a fixed frequency, $f$, and thus represent analogs of the conventional frequency filters that operate at a fixed incidence angle,

\footnotetext{
* Corresponding author.

E-mail address: aeserebr@gmail.com (A.E. Serebryannikov).
}

$\theta$. Spatial filters are also considered from the spatialfrequency filtering perspective [1]. For example, such filters were employed in the analysis of the spatial spectrum, enhancement of the antenna directivity, radar data processing, aerial imaging, and sorting the incoming radiation according to the source location. The known theoretical and experimental performances of the spatial filters include those based on anisotropic (anti-cutoff) media [2], multilayer stacks combined with a prism [3], resonant grating systems [4], metallic grids over a ground plane [5], interference patterns [1], and axisymmetric microstructures [6]. Various photonic crystal (PhC) 
structures that enable efficient spatial filtering should be mentioned, including those based on two-dimensional regular (defect-free) $\mathrm{PhCs}$ and one- and two-dimensional chirped PhCs [7-11]. The co-existing spatial and frequency filtering has also been studied in connection with the control of laser radiation with the aid of the resonant grating based filters [12]. The state-of-the-art of spatial filtering has been reviewed in [13].

The known mechanisms of spatial filtering differ in that they use $[2,7-9,11]$ or do not use $[1,4]$ the peculiar dispersion features. The former may give more freedom in design, because the required features can appear in wide ranges of frequency and/or angle of incidence. For instance, low-pass spatial filtering can be obtained by using volumetric structures with isotropic-type dispersion, which corresponds to the refractive index values within the range of $0<n<1$ [14]. In turn, high-pass and band-pass filtering require structures with anisotropictype dispersion. Generally, these types of spatial filtering can be attained by using anti-cutoff media [2], which are also associated with hyperbolic metamaterials [15] and $\mathrm{PhCs}$ with the dispersion features that enable blocking transmission in the vicinity of zero of tangential wavenumber [7-9]. The problem can appear when a nearly perfect transmission within the entire wide angledomain pass band is required [16,17]. High-pass/dual band-pass spatial filtering that fulfills this requirement has been demonstrated in the transmission mode for a slab of the two-dimensional dielectric PhC [7]. For operation in the reflection mode, high-/band-pass spatial filters have recently been suggested, which are based on two-dimensional $\mathrm{PhCs}$ and single-layer rod arrays over a metallic reflector [18]. Note that the reflection mode high-/band-pass spatial filters require the redistribution of the incident-wave energy in favor of higher diffraction orders, i.e., this mode is connected with the blazing regime [19]. On the contrary, the transmission mode does not require the contribution of higher orders, although blazing, if appears, can lead to some advances in functionality. Regardless of the possible contribution of higher orders, the richness of dispersion features remains the main argument in favor of using two-dimensional PhCs.

In this paper, we demonstrate that a rich variety of the types of spatial filtering, e.g., low-pass, high-/bandpass and bandstop filtering can be realized in one simple configuration that represents a finite-thickness slab of a two-dimensional $\mathrm{PhC}$ composed of circular dielectric rods. The crucial key knobs of the initial design stage that is based on the dispersion analysis include the following: (i) engineering single or multiple Floquet-Bloch waves by having bands with monotonous dispersion which are separated well from each other, bands with nonmonotonous dispersion, or co-existing bands; (ii) engineering the relative position of the modes within the first Brillouin Zone (BZ) with respect to the equifrequency dispersion contours (EFC) in air (for instance, isotropic-type $\mathrm{EFCs}$ of $\mathrm{PhC}$ narrower than in air favor low-pass spatial filtering; anisotropic-type EFCs would favor high-/band-pass spatial filtering); and (iii) engineering the shape of EFCs (e.g., square shape of EFCs is expected to be preferable to keep nearly the same transmission efficiency within the entire band). These points are illuminated by the analysis of the possible combinations of EFCs in air and $\mathrm{PhC}$ and related coupling scenarios. Then, we show by using the simulated transmission results that solely a proper parameter adjustment can enable different types of spatial filtering in the neighboring frequency ranges. To realize a desired response in the angle domain at a fixed frequency, either a sole Floquet-Bloch mode or two such modes are employed. The sharp filter properties are obtained due to the contributions from several effects, such as the shape of the EFCs, Fabry-Perot type interferences, and the excitation of higher diffraction orders. The transmission results have been obtained by using the coupled-integralequation technique, a flexible iterative technique which enables acceleration in convergence by applying preconditioning [20].

\section{Dispersion based analysis}

The basis of operation of spatial filters is connected with the distribution of the Floquet-Bloch modes in the entire wavevector space (not just in the first BZ). It is well known that electromagnetic waves follow in $\mathrm{PhCs}$ the Floquet-Bloch theorem as electrons in a crystal. Therefore, the distribution of the modes in the wavevector space can be reconstructed from the one in the first BZ according to a repeated zone scheme, by following the symmetry of the lattice just like in the electronic case. This approach can simplify both analysis and design significantly. It is noteworthy that an extensive analysis of refraction in PhCs, by taking into account the mode distribution in the entire wavevector space, was first done by Foteinopoulou and Soukoulis [21]. For the purposes of spatial filtering by using $\mathrm{PhCs}$, the results presented in [21] are very important since they illustrate the possible behavior (shape and locations) of the EFCs for PhC in the entire wavevector space, whilst the sign of refraction and handedness are not so important, in the contrast to what might be important for other applications. The same remains true for other previous studies of $\mathrm{PhCs}$, e.g., see $[22,23]$. Comparing to the study of refraction in 
[21], the situation when two beams are simultaneously refracted at the same values of $f$ and $\theta$ is not expected to be useful for spatial filtering. Instead, it is important for us to know which shapes and locations of EFCs for $\mathrm{PhCs}$ are possible.

We restrict our consideration to the case of a squarelattice $\mathrm{PhC}$ and the interfaces of the slab of this $\mathrm{PhC}$ that are along the $\Gamma-\mathrm{X}$ direction. In the band regimes with monotonous dispersion, one Floquet-Bloch wave may couple to the incident and outgoing waves, leading to one transmission band in $\theta$-domain. However, in the band regimes with two band solutions, two Floquet-Bloch modes may be coupled simultaneously, resulting in two transmission bands. Alternatively, one band with a nonmonotonous dispersion can yield two transmission bands in the $\theta$-domain. Taking into account the known richness of dispersion types achievable using $\mathrm{PhCs}$, it is possible to expect obtaining different types of spatial filtering within the neighboring frequency ranges. Here, we assume that either a sole or two Floquet-Bloch modes are used, but the number of the simultaneously utilized modes can formally be arbitrary.

The above given description is depicted in Fig. 1 where typical scenarios of spatial filtering are schematically shown. The EFC shapes used in Fig. 1 are either the same or close to the realistic ones, as follows from the numerous $\mathrm{PhC}$ studies [21-23]. Predictions regarding the existence and the realizable types of spatial filtering are based on the analysis of the shape and location of EFCs. To ensure that a desired coupling regime and related pass band may occur, EFCs for $\mathrm{PhC}$ and the surrounding air must coexist in the corresponding range of variation of the tangential wavevector component, $k_{x}$, which is along the virtual interface, at a fixed frequency. For the sake of definiteness, we assume that the interfaces of the slab of $\mathrm{PhC}$ are along the $x$-direction, see Fig. 2(a). Thus, each $k_{x}$-value for the circular EFC in air unambiguously corresponds to a certain value of $\theta$, i.e., $k_{x}=k \sin \theta$ where $k=\omega / c(\omega=2 \pi f)$ means the radius of this EFC. The dashed vertical lines represent the construction lines in the limiting case, i.e., at the boundaries of $k_{x}$-ranges, in which coupling of the incident wave to a Floquet-Bloch mode is allowed by the dispersion at $k_{x}<0$. Generally, construction lines serve a graphical representation of the conservation of the tangential component of the wavevector, so that each such a line essentially indirectly represents the incidence angle. If a construction line crosses both the EFC for air and the $\mathrm{EFC}$ for $\mathrm{PhC}$, coupling is possible at the chosen values of $\theta$ and $f$.

It is worth noticing the symmetry of EFCs with respect to $k_{x}=0$, so that the pass and stop bands can
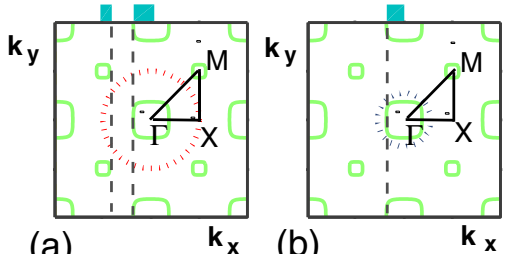

(a)
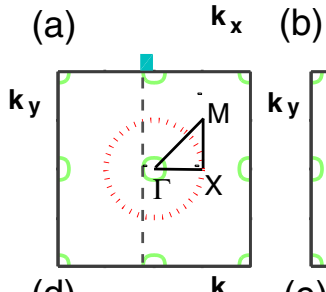

(d)

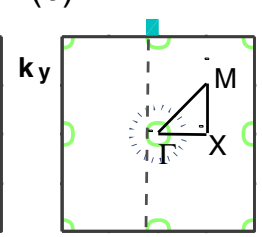

(e)

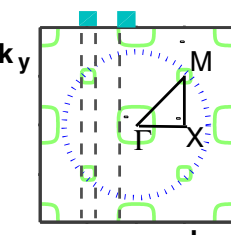

(c)
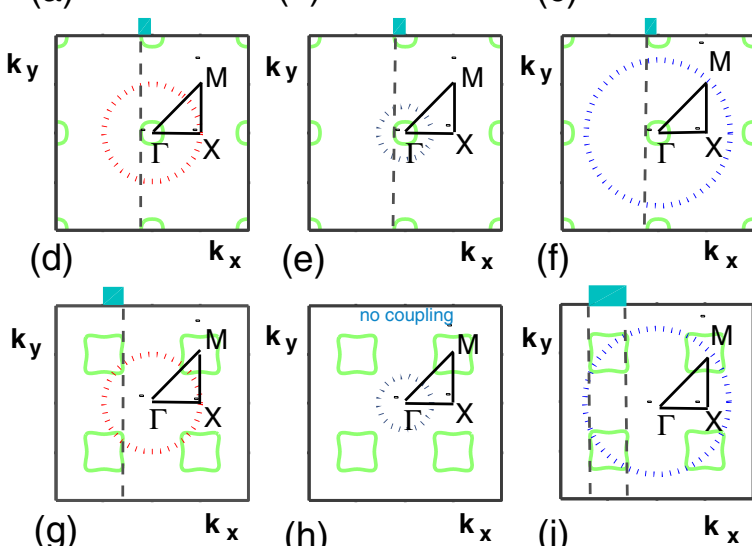

(f)

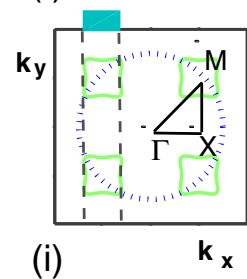

(i)

Fig. 1. (a) Different combinations of mutual location of EFCs for $\mathrm{PhC}$ (solid lines) and air host (dotted lines - circles) in the repeated zone diagram at fixed frequency; components of wavevector, $k_{x}$ and $k_{y}$, are assumed to vary from $-2 \pi / a$ to $2 \pi / a$ ( $a$ is lattice constant); triangles show boundaries of the first BZ; vertical dashed lines - construction lines at the boundaries of the $k_{x}$-ranges where coupling is possible; rectangles schematically show the location of the $k_{x}$-ranges in which coupling is possible at $k_{x}<0$; (a-c) case of two band solutions or one nonmonotonous band solution that lead(s) to two groups of EFCs including those around $\Gamma$-point and $\mathrm{M}$-point of the first BZ, at different values of the EFC radius for air, $k$ : (a) $k=\pi / a$, (b) $k<\pi / a$, and (c) $k>\pi / a$; (d-f) case of one monotonous band solution that leads to one group of EFCs, which are narrower than in air, including an EFC located around $\Gamma$-point of the first BZ: (d) $k=\pi / a$, (e) $k<\pi / a$, and (f) $k>\pi / a ;(\mathrm{g}-\mathrm{i})$ case of one band solution that leads to one group of EFCs including that around M-point of the first BZ: (g) $k=\pi / a$, (h) $k<\pi / a$, and (i) $k>\pi / a$.

appear in the same ranges of variation of $\left|k_{x}\right|$ when $k_{x}>0$ and $k_{x}<0$. Moreover, both ranges can simultaneously be employed. In this case, more pass and stop bands can be obtained in $\theta$-domain at $f=$ const (e.g., dual band-pass filtering instead of single band-pass filtering). However, in this paper, consideration is restricted to the case when $\operatorname{sgn} k_{x}=$ const. Moreover, in this section we assume that higher diffraction orders $(|m|>0)$, which may appear due to the periodicity of the interfaces that is inherited from the square lattice, are not coupled to Floquet-Bloch modes.

In Fig. 1(a-c), the EFCs of the PhC consist of two groups, including those located around $\Gamma$-point and $\mathrm{M}$ point. Such a situation can appear due to two monotonous band solutions [7] or one nonmonotonous band solution [25]. The difference between the plots (a), (b), (c) is 


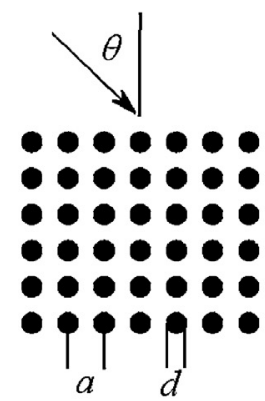

(a)

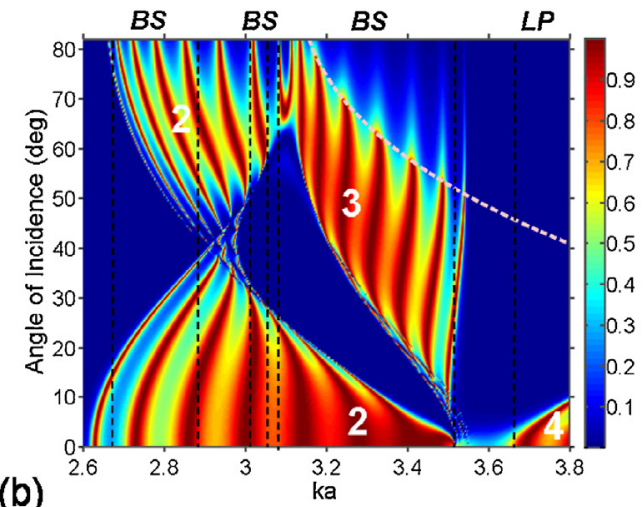

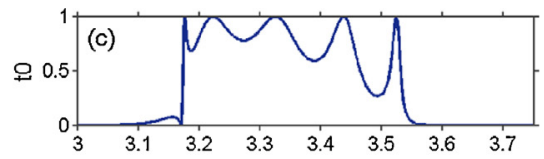
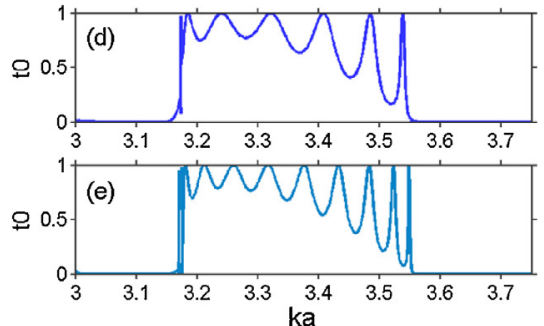

Fig. 2. (a) General geometry used in transmission studies; (b) Map of $t_{0}$ in $(k a, \theta)$-plane at $d / a=0.443, \varepsilon=9.61$, and $N=8$; straight vertical lines - approximate boundaries of $k a$-ranges that can be used for bandstop (BS) and low-pass (LP) spatial filtering, dashed curve - threshold line for the diffraction order $m=-1$; large numbers, 2,3 and 4, indicate transmission area due to the corresponding Floquet-Bloch mode; (c-e) $t_{0}$ vs $k a$ at $d / a=0.443, \varepsilon=9.61, \theta=47^{\circ}$, and (c) $N=6$, (d) $N=8$, and (e) $N=12$.

related to the ratio of the sizes of EFCs for $\mathrm{PhC}$ and air host along the $k_{x}$-axis. In fact, the different radii of EFCs for air mean here different dispersion properties of the PhC and different scaling ratios for the $k_{x}$ and $k_{y}$ axes rather than different properties of the host medium. One can see that bandstop, low-pass, and dual band-pass spatial filtering can be obtained according to Fig. 1(a), (b), and (c), respectively. Next, Fig. 1(d-f) demonstrates the case when the EFCs for PhC include an EFC located around $\Gamma$-point, but are narrower than the $\mathrm{EFC}$ for air. It appears in the case of a sole monotonous band solution. Now, low-pass spatial filtering can only be realized, with the range of the allowed angles, which is determined by the ratio of the widths of EFCs for $\mathrm{PhC}$ and air, i.e., $\max |\theta|=\arcsin \left[\max k_{x}^{\mathrm{PhC}} / k\right]\left(k_{x}^{\mathrm{PhC}}\right.$ is $x$-component of the wavevector in $\mathrm{PhC}$ ). Finally, Fig. 1(g-i) presents the case when the EFCs include an EFC located around Mpoint of the first BZ. This is also possible for a sole band solution. Depending on the width of EFC in air, one can obtain high-pass filtering, perfect reflection, and band-pass filtering as shown in the plots (g), (h), (i), respectively. To conclude, an EFC around $\Gamma$-point is necessary for low-pass filtering, and an EFC around M-point is necessary for high-pass filtering. On the other hand, dual band-pass and bandstop filtering can be obtained when EFCs are located around $\Gamma$-point and M-point; single band-pass filtering requires an $\mathrm{EFC}$ around M-point. Generally, involving higher BZs extends the variety of the achievable scenarios.

\section{Transmission results and discussion}

Geometry of the finite-thickness slab of $\mathrm{PhC}$ is shown in Fig. 2(a). It contains $N$ layers of circular dielectric rods with diameter $d$ and permittivity $\varepsilon$. The rods are placed in a square lattice with constant $a$. The structure is illuminated by $s$-polarized plane wave (electric field is parallel to the rod axes) at the incidence angle $\theta$. The map of zeroorder transmittance, $t_{0}$, is presented in Fig. 2(b) in the $(k a, \theta)$-plane for the slab of $\mathrm{PhC}$, which has been studied theoretically and experimentally at microwave frequencies [8]. The areas of vanishing transmission correspond to incomplete band gaps, i.e., the transmission is blocked for a finite portion of the entire range of $\theta$ variation. Such band gaps are particularly appropriate for spatial filtering, at least if a steep switching between wide pass and stop bands is required. Several ranges that are appropriate for spatial filtering at fixed $f$ can be seen between $k a=2.6$ and $k a=3.8$. These ranges correspond to a sole nonmonotonous band solution or two band solutions. The coupling scenarios realized at $2.6<k a<3.5$ are similar to those illustrated by Fig. 1(a and c). In the areas of strong transmission in Fig. 2(b), there are alternating mountains of nearly perfect transmission and valleys of lower transmission. In fact, they represent different cases of the Fabry-Perot type resonances, including unusual ones. Only those valleys and mountains, which are shifted to larger $k a$ values while $\theta$ is increased, correspond to the conventional Fabry-Perot resonances and, thus, to the case when the effective index of refraction is $n>0$ [24]. On the contrary, those shifting to smaller $k a$ values correspond to the case of $n<0$ [25]. The sign of the effective index is connected to the curvature of the EFCs. Both cases of $n>0$ and $n<0$ can be utilized for the purposes of narrowband spatial filtering. For wideband bandstop and band-pass filtering, the Fabry-Perot resonances are required, which would allow one obtaining mountains of $t_{0} \approx 1$ that are not shifted while varying $\mathrm{ka}$ within a certain $\theta$-range. This should correspond to the cases when the EFC curvature is zero for a certain range 
of $k_{x}$ and, thus, the constant phase condition can be fulfilled for a Fabry-Perot transmission resonance within a desired angle range. Basically, it is possible due to the fact that $k_{y}=$ const for this range. Thus, the closer the EFC shape to square one is, the weaker the sensitivity of the spectral location of the mountain peak to variations in $\theta$ could be. For example, this situation can be realized for the third lowest mode and partially for the second lowest mode at $3.12<k a<3.5$, a range used in our earlier studies of spatial filtering $[7,8]$. In this case, dual band-pass filtering can be obtained. However, the problem remains regarding simultaneously large values of $t_{0}$ for the both large- and small-angle pass bands. On the other hand, the same $k a$ range can be utilized for bandstop filtering regime regardless of the above-mentioned problem.

In fact, the condition of zero curvature of EFCs coincides with that required for finite angle range collimation inside the PhC. Hence, efficient collimation should coexist with spatial filtering that is characterized by $t_{0} \approx 1$ at the mountains. Strictly speaking, these two regimes represent two sides of one phenomenon. In turn, the depth of the valleys and distance between the neighboring mountain peaks depend on the scenario of the evolution of EFCs at varying $\mathrm{ka}$. It can be characterized, for instance, in terms of the effective group index and effective impedance. However, for the purposes of engineering spatial filters with suitable characteristics, it is less useful than the direct analysis of transmission. Thus, the main attention is paid to the effect exerted by variation in $d / a$ on the topology and other features of the transmittance maps in the $(k a, \theta)$-plane.

Let us mention two more interesting features observed in Fig. 2(b). First, in the vicinity of $k a=2.95$ and $\theta=40^{\circ}$, we obtain multiple and very narrow mountain peaks of $t_{0}=1$, which appear for the second lowest mode due to the specific transformation of the EFCs while varying $k a$. This type of behavior, which is possible for a nonmonotonous band solution, has been studied in detail in [25]. Secondly, a semi-ring of $t_{0} \approx 1$ at $3.08<k a<3.12$ and $\theta>65^{\circ}$, which appears due to the second and third lowest modes, should be noticed. Moreover, one should mention low-pass filtering that occurs at $3.65<k a<3.8$ due to the fourth lowest mode, which corresponds to a monotonous band solution. Angular band width is increased here with the value of $k a$ and with the width of the nearly circular EFCs being similar to those in Fig. 1(d-f). Although low-pass spatial filtering is known as an easily obtainable regime, its coexistence with other types of filtering in other frequency ranges but in the same structure can be important for multifunctional operation.
It is noteworthy that $t_{0}$ in Fig. 2(b) dramatically decreases above the first-order threshold line, where redistribution of the incident-wave energy in favor of the diffraction order $m=-1$ takes place. As a result, behavior of $t_{0}$ in the $(k a, \theta)$-plane may differ from that predicted with the aid of EFC analysis, in which possible effects of higher orders are ignored. In fact, one may consider $\theta$-domain behavior at $3.12<k a<3.5$ as either bandstop filtering or dual band-pass filtering. Fig. 2(c-e) presents dependences of $t_{0}$ on $k a$ at the selected value of $\theta$, for different values of $N$. One can see that the transmittance maxima are located within nearly the same $k a$-range, whereas the difference occurs in the number of the peaks of $t_{0}=1$ and valleys and distance between them. The value of $N$ only affects the density, i.e., the number of the mountain peaks and valleys in the same manner as in the classical Fabry-Perot resonators [24,25].

In order to demonstrate that the features observed in Fig. 2(b) are quite general, we vary the PhC parameters. For the transmittance map shown in Fig. 3(a), we take a larger value of $N$ and simultaneously a smaller value of $d / a$. The value of $d / a$ affects location and transmission properties of the areas in $(k a, \theta)$-plane, which are connected with different Floquet-Bloch modes. As expected, they are shifted toward larger $k a$-values for all three modes considered, owing to a smaller value of $d / a$.

An important observation in Fig. 3(a) concerns the fact that now the mountains of $t_{0}$ for the third lowest mode tend to merge, while the valleys become weaker pronounced, see also Fig. 3(d). This leads to rather large areas of high transmission in the $(k a, \theta)$-plane, e.g., at $3.5<k a<3.7$ and $30^{\circ}<\theta<60^{\circ}$. The same and even stronger pronounced effect appears for the fourth lowest mode at $3.85<k a<4.15$, i.e., in the low-pass spatial filtering regime. However, similar but weaker merging that appears for the third lowest mode is more important, because this mode contributes to bandstop/band-pass filtering. It is worth noting the strong transmission owing to the diffraction order $m=-1$ that takes a big part of the incident-wave energy in the area above the threshold line (not shown). A sharp switching between the orders $m=0$ and $m=-1$ is presently under study.

In the contrast to Fig. 2(b), there is a range of band-pass spatial filtering at $3.58<k a<3.83$ in Fig. 3(a), where transmission vanishes at small values of $\theta$, in line with the scenarios that are schematically shown in Fig. 1(g and i). The use of three neighboring $k a$-ranges, e.g., $3.39<k a<3.58,3.58<k a<3.83$ and $3.85<k a<4.15$, enables bandstop/dual band-pass, band-pass, and low-pass spatial filtering, respectively. Thus, the variety of spatial filtering regimes achievable in one simple $\mathrm{PhC}$ based structure can be quite rich. 

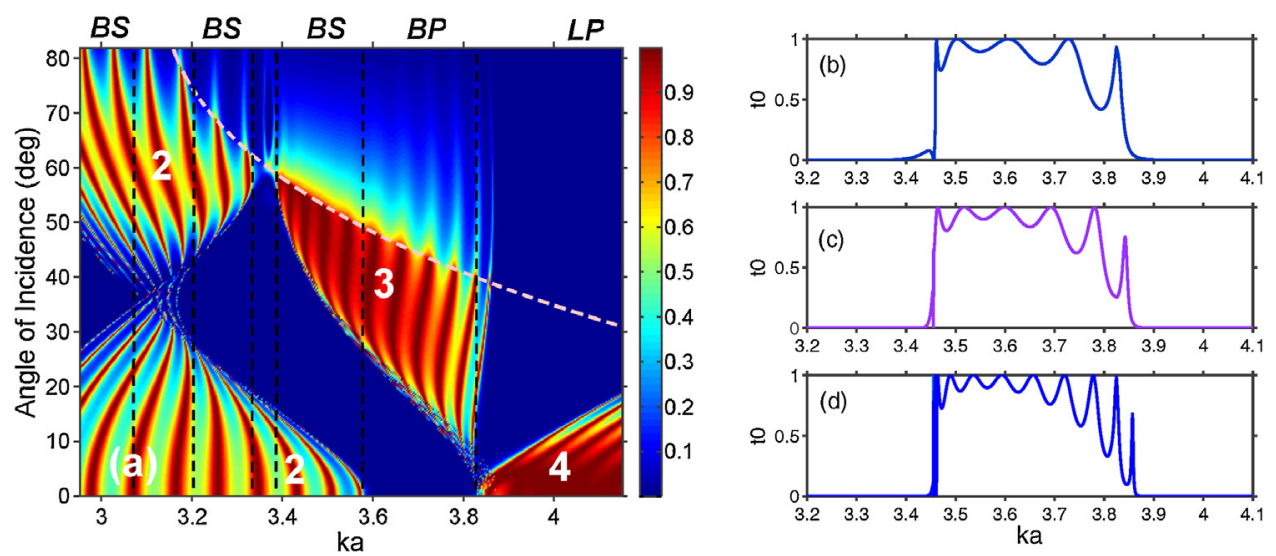

Fig. 3. (a) Map of $t_{0}$ in $(k a, \theta)$-plane at $d / a=0.4, \varepsilon=9.61$, and $N=12$; straight vertical lines - approximate boundaries of $k a$-ranges which can be used for bandstop (BS), band-pass (BP), and low-pass (LP) spatial filtering, dashed curve - threshold line for the diffraction order $m=-1$; large numbers, 2, 3 and 4, indicate transmission area due to the corresponding Floquet-Bloch mode; (b-d) $t_{0}$ vs $k a$ at $d / a=0.4, \varepsilon=9.61, \theta=40^{\circ}$, and (b) $N=6$, (c) $N=8$, and (d) $N=12$.

The second and third of them appear due to the third and fourth lowest modes, respectively, whereas the first one owes to the second and third lowest modes. As in Fig. 2(b), bandstop filtering can also be obtained at $2.95<k a<3.08$ and $3.17<k a<3.33$ due to the second mode corresponding to a nonmonotonous band solution. This regime corresponds to the coupling scenarios shown in the schematics in Fig. 1(a and c). Similarly to Fig. 2(b), the problem of keeping $t_{0}$ high and constant simultaneously within both pass bands can complicate the possible obtaining of efficient wideband dual bandpass filtering at the selected values of $k a$, which belong to the range being appropriate for bandstop filtering. Fig. 3(b-d) demonstrates the effect of $N$ on the dependencies of $t_{0}$ at fixed $\theta$. Again, Fabry-Perot type behavior is evident. Note that $t_{0}<1$ at one of the peaks in these plots because of the effect of the order $m=-1$.
Next, let us further decrease the value of $d / a$. Fig. 4 presents the transmission results for $d / a=0.35$, whereas $N$ is again the same as in Fig. 2(b and d). As far as the role of the order $m=-1$ becomes more important, the transmission results are presented here for the both propagating orders, i.e., $m=0$ and $m=-1$. A wider $k a$ range of single band-pass spatial filtering can be obtained due to zero-order transmission as compared to Fig. 3(a), see Fig. 4(a). It is located now at $3.72<k a<4.2$. As in Fig. 3, this regime may occur here owing to the dispersion behavior like that shown schematically in Fig. 1(g and i). The $(k a, \theta)$-area of high transmission due to the order $m=-1$ is seen in Fig. 4(b). It can be properly combined with the pass bands connected with the order $m=0$ for the same mode. Comparing zero-order transmission for the second lowest mode in Figs. 2(b), 3(a), and 4(a), one can see that not only types of spatial filtering but
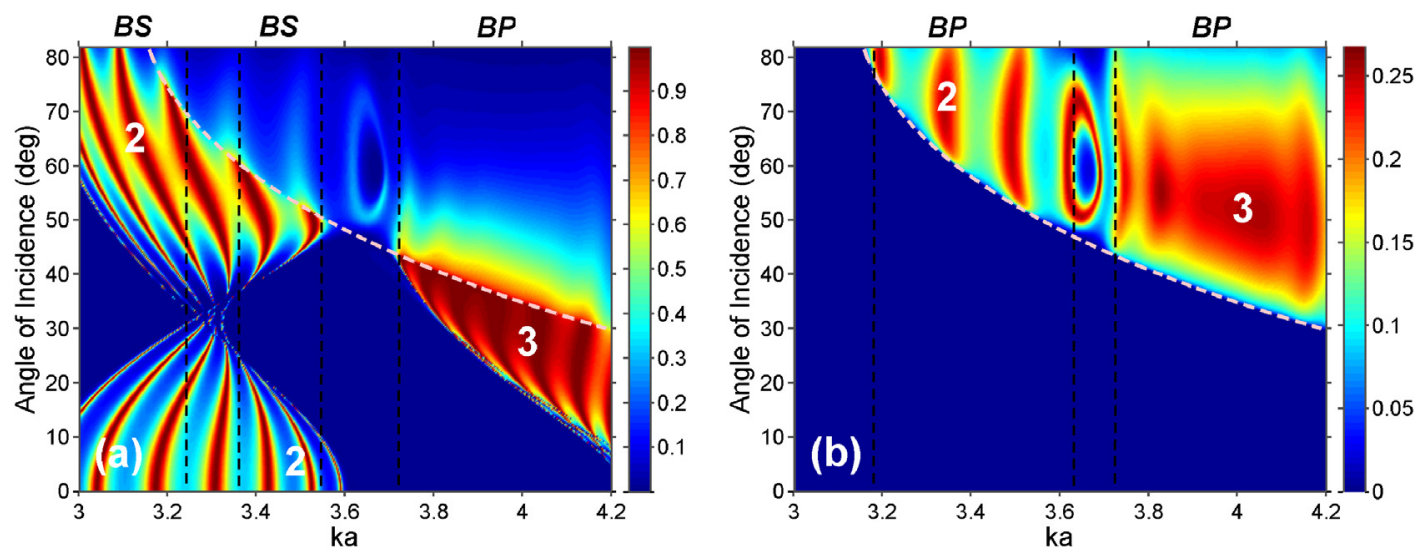

Fig. 4. Maps of (a) $t_{0}$ and (b) $t_{-1}$ in ( $\left.k a, \theta\right)$-plane at $d / a=0.35, \varepsilon=9.61$, and $N=8$; straight vertical lines - approximate boundaries of $k a$-ranges which can be used for bandstop (BS) and band-pass (BP) spatial filtering, dashed curve - threshold line for the order $m=-1$; large numbers, 2 and 3, indicate transmission areas due to the corresponding Floquet-Bloch modes; note that the different scales are used in plots (a) and (b). 

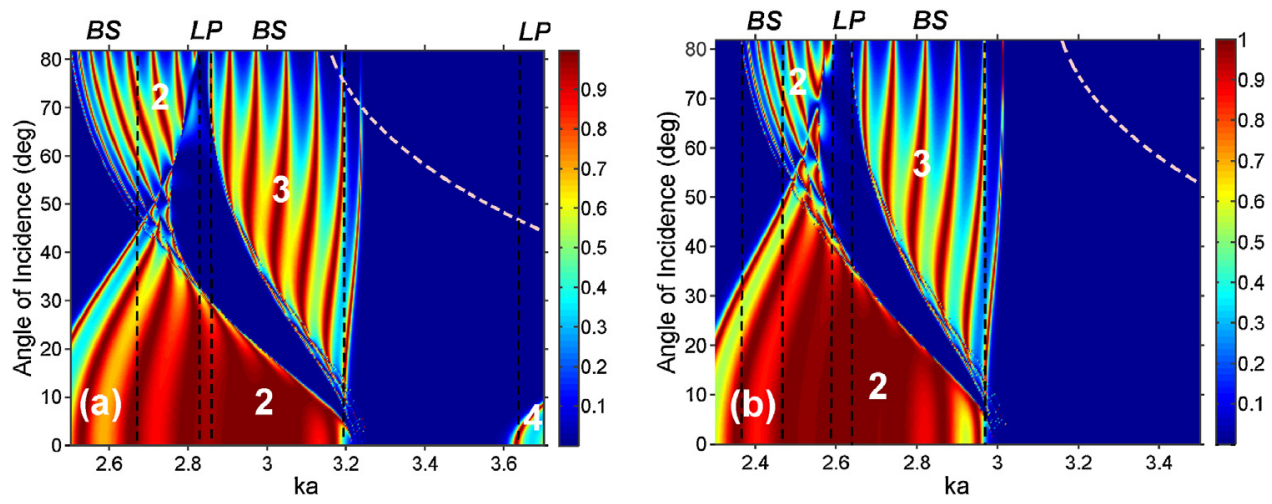

Fig. 5. Maps of $t_{0}$ in $(k a, \theta)$-plane at (a) $d / a=0.5$, (b) $d / a=0.55, \varepsilon=9.61$, and $N=8$; straight vertical lines - approximate boundaries of $k a$-ranges which can be used for bandstop (BS) and low-pass (LP) spatial filtering, dashed curve - threshold line for the order $m=-1$; large numbers, 2 and 3, indicate transmission areas due to the corresponding Floquet-Bloch modes.

also basic characteristics, e.g., locations of transmittance mountains and locations and depths of the valleys can easily be modified just by varying $d / a$.

Zero-order transmission becomes weaker above the threshold line in Fig. 4(b), since the incident-wave energy is redistributed in favor of the order $m=-1$. Among the features observed in Fig. 4(b), the following ones should be noticed: the mountains of $t_{-1}$ that weakly depend on $\theta$ at $k a=3.2, k a=3.35$, and $k a=3.5$; a ring-shaped mountain of $t_{-1}$ that appears near $k a=3.65$; and a wide $(k a, \theta)$-area with gradual variations in $t_{-1}$ that occurs at $3.8<k a<4.2$ and $38^{\circ}<\theta<73^{\circ}$. The first one can be used for multifrequency band-pass spatial filtering at large values of $\theta$. In this case, deflection angle of the outgoing beam of the order $m=-1$ varies from $\phi_{-1}=-77^{\circ}$ at $\theta=64^{\circ}$ to $\phi_{-1}=-65^{\circ}$ at $\theta=75^{\circ}$ when $k a=3.35$, and from $\phi_{-1}=-77^{\circ}$ at $\theta=55^{\circ}$ to $\phi_{-1}=-56^{\circ}$ at $\theta=75^{\circ}$ when $k a=3.5$. The third one is especially appropriate for band-pass filtering when the incident wave has a wide frequency and/or angular spectrum. However, the disadvantage of the use of the order $m=-1$ is that the reflections are quite strong.

Now, we increase $d / a$ ratio compared to the case depicted in Fig. 2(b-e). The results for $d / a=0.5$ and $d / a=0.55$ are presented in Fig. 5(a) and (b), respectively. An area, which might be used for high-/band-pass spatial filtering, is not observed. Just two types of filtering, i.e., low-pass and bandstop filtering, are observed in Fig. 5(a). These regimes require the same shapes and locations of EFCs as similar regimes in Figs. 3 and $4 \mathrm{do}$. At the same time, a new important feature is obtained, which manifests itself in that the individual mountains of $t_{0} \approx 1$ corresponding to the second lowest mode tend to merge at $2.8<k a<3.18$ and $\theta<35^{\circ}$. For example, $t_{0}>0.85$ in the $(k a, \theta)$-plane at $2.63<k a<3.08$ and $\theta<15^{\circ}$. This leads to the appearance of a very large area, which is almost transparent for the incident waves. Hence, this case is particularly appropriate when spatial filtering with a stop band, which is located between two pass bands of a nearly perfect transmission, is required. In particular, it is possible to overcome the difficulties in achieving dual band-pass filtering, which have been mentioned in the analysis of Figs. 2(b) and 3(a). For example, bandstop and dual band-pass regimes can be obtained in Fig. 5(a) at $k a=2.9$, where $t_{0} \approx 1$ for $\theta<25^{\circ}$ and $53^{\circ}<\theta<70^{\circ}$ while $t_{0} \approx 0$ at $27^{\circ}<\theta<51^{\circ}$. Besides, a large area with $t_{0} \approx 0$ occurs in the $(k a, \theta)$-plane at $3.24<k a<3.61$ due to the band gap arising between the third lowest mode and the fourth lowest mode. This results in all-angle separation of the areas of bandstop (leftward) and low-pass (rightward) spatial filtering. At the same time, two mentioned types of spatial filtering can be obtained in one configuration at very close frequencies near $k a=2.83$ due to a very narrow $\theta$-dependent stop band that appears between the transmission areas, which correspond to the second and third lowest modes. However, this low-pass filtering regime enables flexibility in neither the spectral location nor the width of the transmission band in $\theta$-domain.

Fig. 5(b) demonstrates the effect exerted by a further increase of $d / a$ ratio. The main features are the same as in Fig. 5(a). The differences include the shift of the transmission areas connected with the second and third modes toward smaller values of $k a$, widening the stop band adjacent to the lower edge of the transmission area connected with the fourth mode, shift of the area of the strong EFC transformation for the second mode toward larger values of $\theta$ and smaller values of $k a$, and the third lowest mode area becoming fully free of diffraction. Thus, it is evident that not only combinations of various regimes of spatial 
filtering but also other regimes, e.g., the area of strong EFC transformation studied in [25], can be obtained in slabs of $\mathrm{PhC}$ for a wide range of $d / a$ variation.

\section{Conclusion}

To summarize, various regimes of spatial filtering have been studied in simple photonic structures that represent finite-thickness slabs of two-dimensional rod-type photonic crystals (PhCs). Spatial filtering with wide bands of nearly perfect transmission in the incidence-angle domain can be obtained for a wide range of parameter variation. Strictly speaking, one always obtains this or that type of spatial filtering, except for an exotic case when the dispersion for the $\mathrm{PhC}$ is (nearly) the same as that for air. However, obtaining various combinations of the spatial filtering regimes in one configuration that utilize wide angle-domain pass bands with high and nearly constant transmittance needs some parameter adjustments. It has been shown that it can be realized by proper variations in the rod-diameter-tolattice constant ratio, $d / a$, without changing the principal geometrical features of the used structure. Some of the filtering regimes can be strongly affected by blazing, i.e., redistribution of the incident-wave energy in favor of a higher diffraction order. Small values of $d / a$ are well suitable for band-pass spatial filtering. In this case, single band-pass, bandstop, and low-pass filtering can be obtained in the neighboring frequency ranges in one structure that opens a route to multifunctional applications. In turn, large values of $d / a$ are found to be appropriate for bandstop/dual band-pass filtering, with two high-transmittance angle-domain bands at larger and smaller angles that are adjusted to the stop bands arising at intermediate angles. Thus, the obtained results suggest a solution to the problem of two simultaneous angle-domain bands of nearly perfect transmission. It is based on the use of two Floquet-Bloch modes, one of which has the nearly perfect transmission within a large area in the frequency-angle plane. A nearly perfect transmission and related spatial filtering can be achieved at multiple operation frequencies, at least some of which are nearly equidistant from their neighbors. Alongside the variety of dispersion types corresponding to different Floquet-Bloch modes, Fabry-Perot resonances play a very important role in the richness of the achievable regimes of spatial filtering. It has common requirements regarding dispersion and, thus, can co-exist with collimation arising in a finite range of the angles. The presented results may be useful for both traditional and new applications. At the next steps, hybrid regimes will be studied, which include but are not restricted to the combination of collimation, spatial filtering, blazing, and control of coupling strength by corrugations placed at the interface(s).

\section{Acknowledgements}

This work is supported by the projects DPT-HAMIT, ESF-EPIGRAT, and NATO-SET-181 as well as by TUBITAK under Project Nos. 107A004, 109A015, 109E301, and 110T306. The contribution of A.E.S. has partially been supported by TUBITAK in the framework of the Visiting Researcher Programme and by National Science Center of Poland under Project MagnoWa DEC-2-12/07/E/ST3/00538. A.P. acknowledges financial support from the Ministry of Education and Science of Russian Federation in the framework of state task 11.1227.2014/K and from the Government of Russian Federation, Grant 074-U01. E.O. acknowledges partial support from the Turkish Academy of Sciences. The authors are thankful to the anonymous reviewer whose comments allowed to improve this paper.

\section{References}

[1] L. Dettwiller, P. Chavel, Optical spatial frequency filtering using interferences, J. Opt. Soc. Am. A 1 (1984) 18-27.

[2] D. Schurig, D.R. Smith, Spatial filtering using media with indefinite permittivity and permeability tensors, Appl. Phys. Lett. 82 (2003) 2215-2217.

[3] I. Moreno, J.J. Araiza, M. Avedano-Alejo, Thin film spatial filters, Opt. Lett. 30 (2005) 914-916.

[4] A. Sentenac, A.-L. Fehrembach, Angular tolerant resonant grating filters under oblique incidence, J. Opt. Soc. Am. A 22 (2005) 475-480.

[5] O.F. Siddiqui, G. Eleftheriades, Resonant modes in continuous metallic grids over ground and related spatial-filtering applications, J. Appl. Phys. 99 (2006) 083102.

[6] V. Purlys, L. Maigyte, D. Gailevicius, M. Peckus, M. Malinauskas, R. Gadonas, K. Staliunas, Spatial filtering by axisymmetric photonic microstructures, Opt. Lett. 39 (2014) 929-932.

[7] A.E. Serebryannikov, A.Y. Petrov, E. Ozbay, Toward photoniccrystal based spatial filters with wide-angle ranges of total transmission, Appl. Phys. Lett. 94 (2009) 181101.

[8] E. Colak, A.O. Cakmak, A.E. Serebryannikov, E. Ozbay, Spatial filtering using dielectric photonic crystals at beam-type illumination, J. Appl. Phys. 108 (2010) 113106.

[9] R. Pico, I. Perez-Arjona, V.J. Sanchez-Morcillo, K. Staliunas, Evidences of spatial (angular) filtering of sound beams by sonic crystals, Appl. Acoust. 74 (2013) 945-948.

[10] V. Purlys, L. Maigyte, D. Gailevicius, M. Peckus, M. Malinauskas, K. Staliunas, Spatial filtering by chirped photonic crystals, Phys. Rev. A 87 (2013) 033805.

[11] P.V. Usik, A.E. Serebryannikov, E. Ozbay, Spatial and spatialfrequency filtering using one-dimensional graded-index lattices with defects, Opt. Commun. 282 (2009) 4490-4496. 
[12] R. Rabady, I. Avrutsky, Experimental characterization of simultaneous spatial and spectral filtering by an optical resonant filter, Opt. Lett. 29 (2004) 605-607.

[13] L. Maigyte, K. Staliunas, Spatial filtering with photonic crystals, Appl. Phys. Rev. 2 (2015) 011102.

[14] B.T. Schwartz, R. Piestun, Total external reflection from metamaterials with ultralow refractive index, J. Opt. Soc. Am. B 20 (2003) 2448-2453.

[15] C. Rizza, A. Ciattoni, E. Spinozzi, L. Colombo, Terahertz active spatial filtering through optically tunable hyperbolic metamaterials, Opt. Lett. 37 (2012) 3345-3347.

[16] E.H. Cho, H.-S. Kim, B.-H. Cheong, O. Prudnikov, W. Xianyua, J.-S. Sohn, D.-J. Ma, H.-J. Choi, N.-C. Park, Y.-P. Park, Twodimensional photonic crystal color filter development, Opt. Express 17 (2009) 8621-8629.

[17] B.-H. Cheong, O.N. Prudnikov, E. Cho, H.-S. Kim, J. Yu, J.-S. Cho, H.-J. Choi, S.T. Shin, High angular tolerant color filter using subwavelength grating, Appl. Phys. Lett. 94 (2009) 213104.

[18] A.E. Serebryannikov, Ph. Lalanne, A.Yu. Petrov, E. Ozbay, Wideangle reflection-mode spatial filtering and splitting with photonic crystal gratings and single-layer rod gratings, Opt. Lett. 39 (2014) 6193-6196.

[19] A. Hessel, J. Schmoys, D.Y. Tseng, Bragg-angle blazing of diffraction gratings, J. Opt. Soc. Am. 65 (1975) 380-384.

[20] T. Magath, A.E. Serebryannikov, Fast iterative, coupled-integralequation technique for inhomogeneous profiled and periodic slabs, J. Opt. Soc. Am. A 22 (2005) 2405-2418.

[21] S. Foteinopoulou, C.M. Soukoulis, Electromagnetic wave propagation in two-dimensional photonic crystals: a study of anomalous refractive effects, Phys. Rev. B 72 (2005) 165112.

[22] A.E. Serebryannikov, One-way diffraction effects in photonic crystal gratings made of isotropic materials, Phys. Rev. B 80 (2009) 155117

[23] C. Luo, S.G. Johnson, J.D. Joannopoulos, J.B. Pendry, All-angle negative refraction without negative effective index, Phys. Rev. B 65 (2002) 201104(R).

[24] M. Born, E. Wolf, Principles of Optics, 4th ed., Pergamon Press, Oxford, 1970.

[25] A.E. Serebryannikov, E. Ozbay, P.V. Usik, Defect-mode-like transmission and localization of light in photonic crystals without defects, Phys. Rev. B 82 (2010) 165131. 